\title{
AUTOMATIC TEXT CLASSIFICATION USING AN ARTIFICIAL NEURAL NETWORK
}

\author{
Rodrigo Fernandes de Mello \\ Universidade de São Paulo \\ Instituto de Ciências Matemáticas e de Computação \\ Departamento de Ciências da Computação e Estatística \\ Caixa Postal 668 \\ 13560-970 São Carlos - SP \\ mello@icmc.usp.br
}

\section{Luciano José Senger}

Universidade Estadual de Ponta Grossa

Departamento de Informática

Av. Carlos Cavalcanti, 4748

84030-900 Ponta Grossa-PR

ljsenger@icmc.usp.br

\section{Laurence Tianruo Yang}

Department of Computer Science

St. Francis Xavier University

Antigonish, NS, Canada

lyang@stfx.ca

\begin{abstract}
The increasing volume of available documents in the World Wide Web has turned the document indexing and searching more and more complex. This issue has motivated the development of several researches in the text classification area. However, the techniques resulting from these researches require human intervention to choose the more adequate parameters to carry on the classification. Motivated by such limitation, this article presents a new model for the text automatic classification ${ }^{1}$. This model uses a self-organizing artificial neural network architecture, which does not require previous knowledge on the domains to be classified. The document features, in a word radical frequency format, are submitted to such architecture, what generates clusters with similar sets of documents. The model deals with stages of feature extraction, classification, labeling and indexing of documents for searching purposes. The classification stage, receives the radical frequency vectors, submit them to the ART-2A neural network that classifies them and stores the patterns in clusters, based on their similarity
\end{abstract}


level. The labeling stage is responsible for extracting the significance level of each radical for each generated cluster. Such significances are used to index the documents, providing support to the next stage, which comprehends the document searching. The main contributions provided by the proposed model are: proposal for distance measures to automate the $\rho$ vigilance parameter responsible for the classification quality, thus eliminating the need of human intervention on the parameterization process; proposal for a labeling algorithm that extracts the significance level of each word for each cluster generated by the neural network; and the creation of an automated classification methodology.

Keywords: text classifying, neural networks, adaptive resonance theory.

\section{Introduction}

Every day, millions of documents are added to the World Wide Web Pierre, 2000; Sinka and Corne, 2002. This increasing volume of available documents makes the searching operations more and more complex. Nowadays, the search engines use techniques based on the search of word sets within the Web documents. Such techniques spend a lot of computing processes as they require the indexing of all words contained on the documents and consider as of the same importance all the words contained there. Observing the complexity of the document search operations, several researches for text and document classification have been proposed Sinka and Corne, 2002; Pierre, 2000; He et al., 2003; Nigam et al., 2000; Blei et al., 2002. Out of these works, the following ones may be highlighted: M.P. Sinka e D.W. Cornei Sinka and Corne, 2002, J. He et al. He et al., 2003, K. Nigam et al. Nigam et al., 2000 and D.M. Blei et al. Blei et al., 2002. M.P. Sinka and D.W. Cornei Sinka and Corne, 2002 have proposed a pattern data set to accomplish the text classification studies. Such data set is based on html pages on 11 different subjects. Details on this data set are presented, in addition to the results that use the $k$-means algorithm to classify the documents. In order to use this algorithm it was necessary to count the word frequency on each document. For each document it was created a vector containing from $0.05 \%$ to $2 \%$ of the most frequent words. The created vector set was submitted to the $k$-means algorithm, which was responsible for calculating the minimum Euclidean distance among them. With the experiments, it could be proved that the documents average classification rate was above $50 \%$, taking into account the previous classification as a pattern. On the experiments, the $k$-means algorithm was manually parameterized until the desired results were reached.

J. He et al. He et al., 2003 have proposed an alteration to the ART-2A artificial neural network to create a pre-defined number of clusters on the pattern classification. For this purpose, it was proposed a dynamic change on the $\rho$ vigilance parameter, which may fit itself for the creation of a desired number of clusters. This technique is compared to the original ART-2A neural network, 
$\mathrm{SOM}$ and to the batch $k$-means and online $k$-means clustering algorithms. The text classification results have proved that the change on the ART-2A neural network brings about, on certain instances, some improvements on the final results. The major contribution of this work is to allow the previous definition of the desired number of clusters by the end of a classification process.

K. Nigam et al. Nigam et al., 2000 have shown that the classification quality may be increased by means of a small set of documents previously labeled. It has been presenting a learning algorithm that uses labeled and unlabeled documents based on the combined techniques of Expectation-Maximization and a naive Bayes classifier. Before performing classifications with the algorithm, it is required to train the classifier with a subset of labeled documents. The knowledge obtained on this stage is then used to classify the remainder documents. Experiments have shown that errors on unlabeled documents decrease up to $30 \%$.

D.M. Blei et al. Blei et al., 2002 have proposed a classification model that analyzes the global and local features of the documents. The global features comprehend the words and their frequencies; the local features are composed by the font size and color, italic, bold and other components related to the text format. Experiments have been carried out by using this model over a subset of documents for learning purposes and then on the remainder documents to classify them out. The experiment results have proved that classification errors are reduced in about $66 \%$.

The previously presented work have shown good results but the choice of the best parameters is made by human intervention. For instance, M.P. Sinka and D.W. Cornei Sinka and Corne, 2002 have defined the $k$ parameter, from $k$-means algorithm, fixed on 2, J. He et al. He et al., 2003 have defined a fixed number of clusters to be generated by the end of the classification process, what influences the neural network vigilance parameter and may deteriorate the classification quality. K. Nigam et al. Nigam et al., 2000 have shown that the classification quality may be enlarged by submitting a subset of labeled documents to the clustering algorithm. Nevertheless, this technique requires a previous human classification on part of the documents. D.M. Blei et al. Blei et al., 2002 have shown that the classification quality may be improved by considering the local features of each document. However, this technique requires previous training so that the model may learn the different manners to classify the remainder documents.

Observing the previously described limitations, this article presents a new model for automatic text classification. The documents may be stored in any format that may be transformed in pure text, such as html, pdf, postscript, Microsoft Word, Microsoft Excel, etc. This model is composed of the following stages: conversion of document format into pure text, stopwords removal, extraction of word radicals contained on the text, radical frequency counting, 
frequency submission to an ART-2A self-organizing artificial neural network which is responsible for classifying the document and extracting the radicals and their respective significances. Such radicals and significances are used to index the documents. From the keywords typed by the user, searches may be done on the radicals that index the documents, by arranging the result according to its significances. Thus, it is obtained,in a relevance order, the document set that best fits the user's request.

\section{Model for Text Classification}

This work has been motivated by the need to use human intervention to define the classification parameters on clustering algorithms such as batch $k$ means, online k-means and neural network architectures such as SOM and $A R T$. This kind of parameterization has limited the automatic classification application over the unknown dominions. Such limitations have motivated the proposal for an automatic model for text classification, without the need of previous knowledge on the classification dominions and without human interference. The stages that make up this model are described on the following sections.

\section{Feature extraction}

The first stage of the automatic classification model is composed of the preprocessing and feature extraction, which provide subsidies for the classification start up. The steps that make it up are described as follows:

1 Conversion from document format to pure text - on this stage the documents are converted into pure text, so that the word occurrences may be counted on each document. The quantity of occurrences of a same word at a certain document, called $\alpha$ word frequency on the document, is used as the classifier entry.

2 Stopwords Removal- after the document is converted, the words that do not show relevant significance are removed. Examples of such words are: of, the, in, and, or etc. Thus, only the most relevant words are left to represent the document.

3 Extraction of word radicals contained in the text - with the main words of each document it is started the extraction of word radicals, responsible for attributing meaning to them. For instance, words such as balancing, balance, balanced are unified through the balanc radical.

4 Radical frequency counting - after the radicals are extracted, they are counted and the number of occurrences is stored at a distinct vector for each document. Such vectors contain the frequency of each word at a 
certain document. Each $V t_{i}$ vector(where $i=0,1,2, \ldots, n$ ) shows the same number of elements, where $\left|V t_{1}\right|=k,\left|V t_{2}\right|=k, \ldots,\left|V t_{n}\right|=k$. Elements of a same index quantify the frequency of a same word. Thus, an $\alpha$ word is at the same $j$ index of the $V t_{1}, V t_{2}, \ldots, V t_{n}$ vectors.

\section{Classification}

The second stage is responsible for grouping and classifying the $V t_{i}$ vector sets (obtained on the first stage) by using an ART-2A Carpenter et al., 1991 self-organizing artificial neural network architecture.

The ART (Adaptive Resonance Theory) neural network family are selforganizing and non-supervised architectures that learn on stable representation codes in response to an arbitrary sequence of input patterns Carpenter and Grossberg, 1988; Carpenter and Grossberg, 1989. The ability of the ART-2A network family differs from the other self-organizing architectures as it allows the user to control the similarity degree among the patterns grouped at a same representation unit. This control allows the network to be sensitive to the differences existing on the input patterns and to be able to generate more or less classes in response to this control. Moreover, the learning on the networks ART is continuous: the network adapts itself to the incoming data, creating new processing units to learn the patterns, when required. Out of the different versions of networks ART, the architecture ART-2A may be highlighted as it allows the quick learning of the input patterns represented by continuous values. Because of its attractive features, such as noise filtering and good computing and classification performance, the neural ART network family has been used in several domains, such as to recognize Chinese characters Gan and Lua, 1992, interpretation of data originated on nuclear reactor sensors Whiteley and Davis, 1996; Whiteley and Davis, 1993; Keyvan and Rabelo, 1992, image processing Vlajic and Card, 2001, detection of earth mines Filippidis et al., 1999, treatment of satellite images Carpenter et al., 1997 and robots sensorial control Bachelder et al., 1993.

The ART 2A network architecture is composed of two main components: the attention and orientation systems (figure 1). The attention system is provided with an $F_{0}$ preprocessing layer, an $F_{1}$ input representation layer and with a $F_{2}$ class representation layer. The input and representation layers are interconnected through a set of adaptive weights called bottom-up $\left(F_{1} \rightarrow F_{2}\right)$ and top-down $\left(F_{2} \rightarrow F_{1}\right)$. The path from the neuron $i$ th of layer $F_{1}$ to the neuron $j$ th of the layer $F_{2}$ is represented by $w_{i j}$. Likewise, the neuron $j$ th of layer $F_{2}$ is connected to $i$ th of the layer $F_{1}$ through the adaptive weight $w_{j i}$. These weights multiply the signals that are sent among the neuron layers and are responsible for the storage of the knowledge obtained by the network. The interactions between the layers $F_{1}$ and $F_{2}$ are controlled by the orientation 
system, which uses a $\rho$ vigilance parameter, and the way through which the weights are updated to obtain knowledge on the input patterns is defined by the training algorithm.

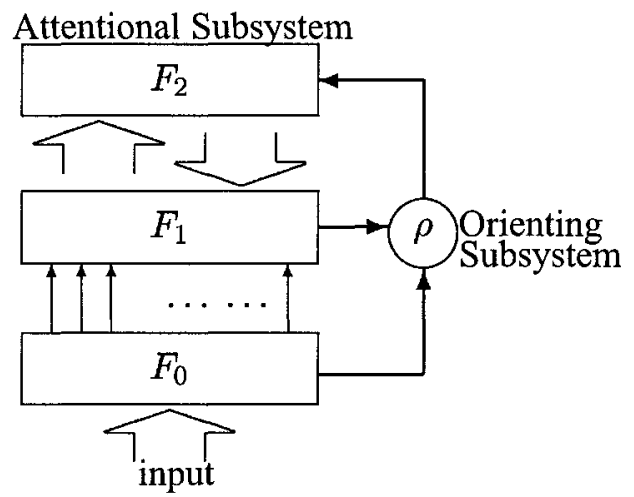

Figure 1. ART 2A neural network basic architecture

Training algorithm. The ART-2A dynamics are determined by the $\rho \in$ $[0,1]$ vigilance parameter and the $\beta \in[0,1]$ learning rate. Initially, the output layer $F_{2}$ does not have any class. On our classification model, the input pattern $I^{0}$ is composed of a set of attributes associated to the process to be classified. The ART-2A training algorithm is composed of the following stages: preprocessing, activation, search, resonance or reset and adaptation.

- Preprocessing: this phase performs input normalization operations $I^{0}$ :

$$
I=\aleph\left(F_{0}\left(\aleph\left(V t^{0}\right)\right)\right)
$$

where $\aleph$ and $F_{0}$ describe the following operations:

$$
\aleph(x) \equiv \frac{x}{\|x\|} \equiv \frac{x}{\sum_{i=0}^{n} x_{i}^{2}}, F_{0}(x)= \begin{cases}x & \text { if } x>\theta \\ 0 & \text { otherwise }\end{cases}
$$

Such operations perform the Euclidean normalization and noise filtering. The noise filtering, through the $\theta$ parameter, only makes sense if the main features of the input patterns, which lead to the creation of different classes, are represented exclusively on the highest values of the input components. 
- Activation: this phase is responsible for sending out the incoming signals to the neurons of the representation layer $F_{2}$ :

$$
T_{j}= \begin{cases}I w_{i j} & \text { if } j \text { indexes a committed prototype } \\ \alpha \sum_{j} I_{j} & \text { otherwise }\end{cases}
$$

where $T_{j}$ corresponds to the $j$ neuron activation on the $F_{2}$ layer. Initially, all the neurons are marked as uncommitted and become committed when their weights are adapted to learn a certain input pattern. The $\alpha$ choice parameter defines the maximum depth of search for a fitting cluster. With $\alpha=0$, value used in this work, all committed prototypes are checked before an uncommitted prototype is chosen as winner.

- Search: This phase is responsible for finding a candidate neuron to store the current pattern. The network competitive learning indicates the most activated neuron is the one chosen as candidate to represent the input pattern:

$$
T_{J}=\max \left\{T_{j}: \text { for all } F_{2} \text { nodes }\right\}
$$

- Resonance or reset: after selecting the most activated neuron, the reset condition is tested:

$$
y_{J}>\rho
$$

If the inequality is real, the candidate neuron is chosen to store the pattern and the adaptation stage is initiated (resonance). If not, the winning neuron is inhibited and the searching stage is repeated (reset).

- Adaptation: this stage describes how the pattern will be learned by the network. Such stage comprehends the updating of the network weights for the $J$ winning neuron, which, then, become committed:

$$
w_{J i}^{n e w}= \begin{cases}\aleph\left(\beta \aleph \Psi+(1-\beta) w_{J i}^{o l d}\right) & \text { if } j \text { indexes a committed prototype } \\ I & \text { otherwise }\end{cases}
$$

$$
\Psi_{i} \equiv \begin{cases}I_{i} & \text { if } w_{J i}^{\text {old }}>\theta \\ 0 & \text { otherwise }\end{cases}
$$


Table 1. ART-2A main parameters

\begin{tabular}{clc}
\hline Parameter & Description & Value example \\
\hline$m$ & number of input units & 7 \\
$n$ & maximum number of representation units & 15 \\
$\theta$ & noise suppression parameter & $\theta=\frac{1}{\sqrt{m}}$ \\
$\beta$ & learning rate & $\beta=0.7$ \\
$\rho$ & vigilance parameter & $\rho=0.9$ \\
\hline
\end{tabular}

The table 1 illustrates the examples of values for the ART-2A network parameters. The $\rho$ vigilance value defines the quantity of classes that will be created by the network. The $\rho$ value forms a circular decision boundary with a radius of $\sqrt{2(1-\rho)}$ around the weight vector of each category He et al., 2003. With $\rho=0$, all input patterns are grouped at a same class. With $\rho=1$, it is created a class for each input pattern presented to the network. The $\beta$ learning rate defines the adaptation speed of the prototypes in response to the input patterns. The ART-2A should not be used with $\beta \cong 1$, as the prototypes tend to jump among the input patterns associated to a class instead of converging to the patterns average.

Each committed neuron of the $F_{2}$ layer defines a similar pattern group. The committed neuron set defines the classification generated by the network for the submitted values. As the input patterns over the process behavior are not previously labeled, it is required an algorithm to define a label to represent each class created by the network.

Labeling Algorithm. The labeling algorithm is built in accordance with the idea that the ART-2A network weights resemble the input patterns that have been learned by a certain neuron of the $F_{2}$ layer Senger et al., 2004. The ART-2A network weights are also called prototypes because they define the direction for the data grouping. The data normalization operations performed by the ART network allows all the vectors to be canonically normalized. Thus, only the angle formed among the prototypes is preserved. If the process monitoring values are initially normalized (see equation 2 ), such values do not differ too much in their magnitude. In addition, according to the rule for updating the ART network weights (see equation 7), the prototypes are also normalized. Thus, each attribute contribution can be obtained, based on its value on the weight vector. Such value represents the attribute significance for the local grouping chosen by the network.

After the data grouping is performed by the ART-2A network, a label is added to each neuron of the $F_{2}$ layer. For this purpose, a significance matrix is defined, which is composed of a $S V_{i j}$ significance value set Ultsch, 1993. The significance matrix supports the decision about which components of the input 
vector are significant to label each committed neuron of the $F_{2}$ layer. The significance values are obtained directly from the ART-2A network weights, where the number of columns of the significance matrix is equal to the number of committed neurons of the $F_{2}$ layer, which represent the obtained classes and the number of lines is equal to the number of components of the input vector. For instance, a $S M=\left(S V_{i j}\right)^{7 \times 4}$ significance matrix is obtained through a network that has 4 classes to represent a process described by an input vector with $m=7$ components.

The labeling algorithm is illustrated by the Algorithm 1. In order to detect the most important attributes to describe the class, the significance values of the attributes are normalized in relation to the sum of the total significance values of a certain class, that is, the sum of the elements of the column. After such normalization is done, the column values are arranged in a decreasing manner and accumulated frequency of the significance values is calculated. For labeling the class, the set of the more significant attributes is selected until the accumulated frequency sum does not exceed a certain $\chi$ threshold. By the end of the algorithm execution there will be a $C$ set of more relevant attributes, for each category created by the network, to label the class.

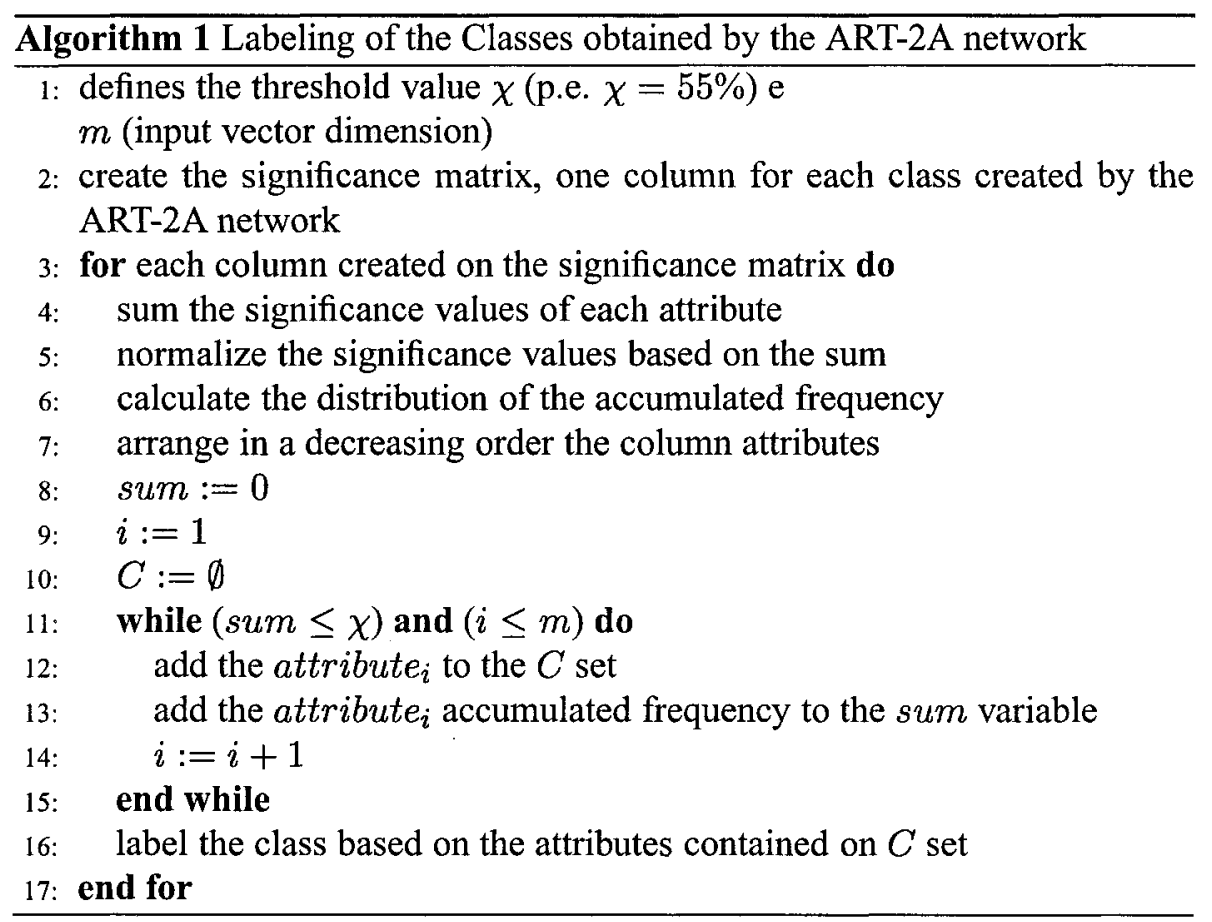

The labeling stage obtains a $C$ attribute set to represent the group. The elements of this set are afterwards used to index the documents. 


\section{Document Indexing and Search}

The $C$ attribute set, obtained through the labeling algorithm, is used to index the documents according to the significance of each word radical for one of the generated clusters.

After the indexing process, searches may be conducted from the keywords typed by the users. Out of these words are extracted the radicals, which are compared to the $C$ attribute set that index the documents. By following this strategy, it may be obtained, in a relevance order, the document set that best fits the user's request.

\section{Example on Text Classification}

An example on text classification is presented on this section. Consider the table 2 as the word radical frequency counting of 10 distinct documents. The radical frequency set of each document is submitted to the ART-2A selforganizing neural network architecture, which is responsible for classifying it and creating clusters based on the document similarity.

Table 2. Radical frequency on Documents

\begin{tabular}{cccccc}
\hline Documents & finance & sport & biology & program & internet \\
\hline \hline 1 & 10 & 5 & 7 & 0 & 1 \\
\hline 2 & 0 & 1 & 15 & 10 & 7 \\
\hline 3 & 2 & 0 & 19 & 5 & 3 \\
\hline 4 & 3 & 7 & 8 & 8 & 5 \\
\hline 5 & 1 & 9 & 3 & 0 & 10 \\
\hline 6 & 8 & 15 & 2 & 9 & 11 \\
\hline 7 & 2 & 1 & 10 & 12 & 12 \\
\hline 8 & 1 & 0 & 8 & 2 & 1 \\
\hline 9 & 10 & 0 & 7 & 7 & 5 \\
\hline 10 & 0 & 12 & 0 & 9 & 3 \\
\hline
\end{tabular}

The number of created clusters and stored patterns on each cluster varies according to the $\rho$ vigilance parameter. The table 3 shows the quantity of clusters and the main word radicals, with their respective percentage significances to $\rho \in[0,1]$.

On the table 3 it may be observed that high $\rho$ values makes the neural network to generate a higher number of clusters. The column Identifiers Set and Significance of this table shows the identifier set and its significances for each created cluster. It may be noted that for the $\rho \in[0.05,0.30]$ it is generated only a set of identifiers, that is, only a cluster is created. For $\rho \in[0.35,0.40]$ are created two clusters and so on. The higher is the number of clusters, the more specific they get and may even reach the limit of containing only one pattern. 
Table 3. Radical Significance for each Cluster

\begin{tabular}{|c|c|c|}
\hline$\rho$ & Clusters & Set of Identifiers and Significance (\%) \\
\hline 0.05 to 0.30 & 1 & \{finance $=43.47$, biology $=30.42\}$ \\
\hline 0.35 to 0.40 & 2 & finance $=43.47$, biology $=30.42\},\{$ sport $=50.00$, program $=37.50\}$ \\
\hline 0.45 & 2 & \{finance $=43.47$, biology $=30.42\},\{$ program $=32.44$, internet $=32.44\}$ \\
\hline 0.50 to 0.65 & 3 & $\begin{array}{l}\text { finance }=43.47, \text { biology }=30.42\},\{\text { biology }=45.48, \text { program }=30.28\} \\
\{\text { internet }=43.51, \text { sport }=39.12\}\end{array}$ \\
\hline 0.70 to 0.75 & 3 & $\begin{array}{l}\{\text { inance }=43.47, \text { biology }=30.42\},\{\text { biology }=45.48, \text { program }=30.28\} \\
\{\text { internet }=43.51, \text { sport }=39.12\},\{\text { sport }=50.00, \text { program }=37.50\}\end{array}$ \\
\hline 0.8 & 4 & $\begin{array}{l}\text { finance }=43.47, \text { biology }=30.42\},\{\text { biology }=45.48, \text { program }=30.28\} \\
\{\text { internet }=43.51, \text { sport }=39.12\}, \text { finance }=34.48, \text { biology }=24.13\}, \\
\{\text { sport }=50.00, \text { program }=37.50\}\end{array}$ \\
\hline 0.85 & 6 & $\begin{array}{l}\{\text { finance }=43.47, \text { biology }=30.42\},\{\text { biology }=45.48, \text { program }=30.28\} \\
\{\text { internet }=43.51, \text { sport }=39.12\},\{\text { sport }=33.31, \text { internet }=24.41\}, \\
\text { \{inance }=34.48, \text { biology }=24.13\}\end{array}$ \\
\hline 0.9 & 7 & $\begin{array}{l}\text { finance }=43.47, \text { biology }=30.42\},\{\text { biology }=45.48, \text { program }=30.28\} \\
\{\text { biology }=25.80, \text { program }=25.80, \text { sport }=22.57\} \\
\{\text { internet }=43.51, \text { sport }=39.12\},\{\text { sport }=33.31, \text { internet }=24.41\} \\
\{\text { inance }=34.48, \text { biology }=24.13\},\{\text { sport }=50.00, \text { program }=37.50\}\end{array}$ \\
\hline 0.95 & 8 & $\begin{array}{l}\{\text { finance }=43.47, \text { biology }=30.42\},\{\text { biology }=45.48, \text { program }=30.28\}, \\
\{\text { biology }=65.54\},\{\text { biology }=25.80, \text { program }=25.80, \text { sport }=22.57\}, \\
\{\text { internet }=43.51, \text { sport }=39.12\},\{\text { sport }=33.31, \text { internet }=24.41\}, \\
\{\text { program }=32.44, \text { internet }=32.44\},\{\text { finance }=34.48, \text { biology }=24.13\}, \\
\{\text { sport }=50.00, \text { program }=37.50\}\end{array}$ \\
\hline
\end{tabular}

As the aim of the neural network is to classify similar patterns in a same cluster, extreme cases where there is only one pattern per cluster get undesirable. It appears, then, the issue of how to define the best value for $\rho$. In order to define its ideal value, this article proposes two performance measures that list the distances among patterns contained in each cluster, and the distance among centroids from distinct clusters He et al., 2003; Gokcay and Principe, 2000.

The first proposed measure is the intra-cluster distance defined by the equation 8. This measure, based on He et al., 2003, allows the quantification of the average distance among the patterns of a cluster and its $c_{j}$ centroid. This measure calculates the average cosine of a $\theta_{i, j}$ angle among the $v_{i, j}$ vectors and the cluster $c_{j}$ centroid, according to the cosine law Pappas, 1989.

$$
\text { Intra }=\frac{\sum_{j=1}^{n c} \sum_{i=1}^{n}\left\|v_{i, j}\right\| *\left\|c_{j}\right\|}{n c}
$$

where $n c$ is number of clusters in the ART- $2 \mathrm{~A} F_{2}$ layer. The second measure, based on Gokcay and Principe, 2000, is the inter-cluster distance defined by the equation 9 , which calculates the average distance among the centroids of clusters generated by the neural network. This equation, as well as the equation 


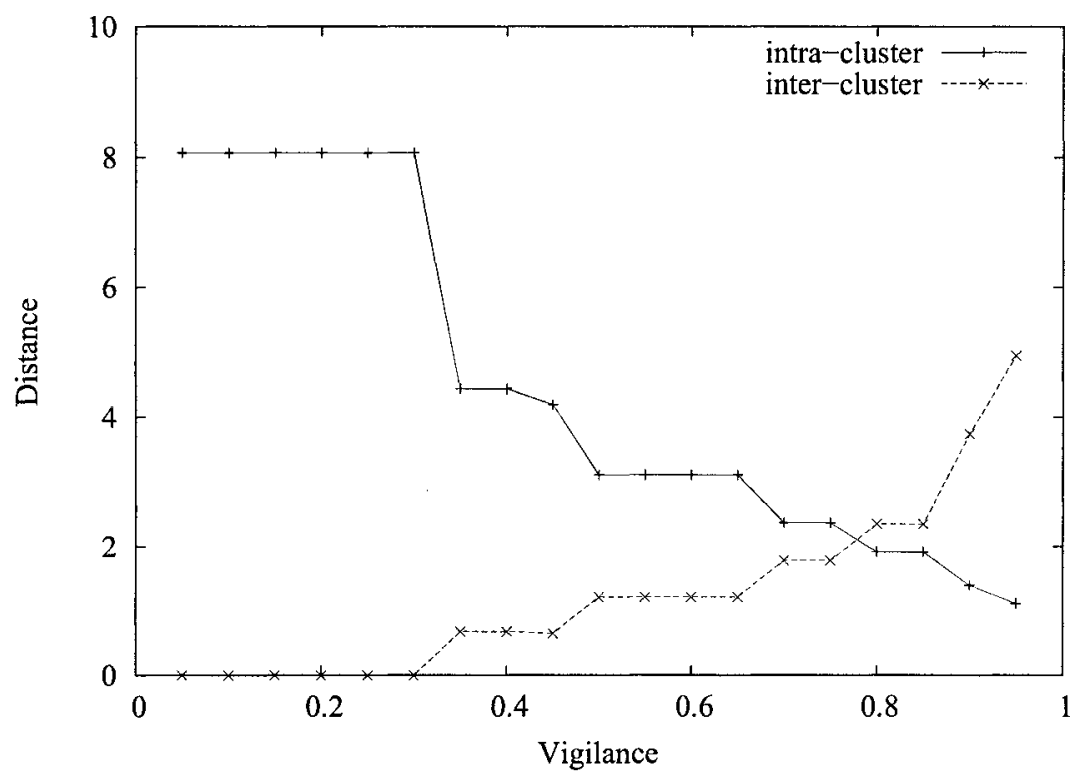

Figure 2. Experiment example: Variance of the intra and inter-cluster distance

8 , also uses the cosine laws. Thus, the results of both equations are generated over a same scale, providing the definition of a point on the $R^{2}$ level where both functions get equal. This point defines the ideal $\rho$ and, consequently, the number of clusters and patterns per cluster generated by the neural network.

$$
\text { Inter }=\frac{\sum_{i=1}^{n c} \sum_{j=1}^{n c}\left\|c_{i}\right\| *\left\|c_{j}\right\|}{n c}
$$

The figure 2 shows the infra-cluster function and the inter-clusters for $\rho \in$ $[0,1]$. Through this figure it may be observed that the functions cross one another to $\rho \in[0.7,0.75]$. On this point, it may be concluded that the intracluster distance is short enough to keep similar patterns at a same cluster, as well as the inter-cluster distance is long enough to separate the clusters created by the neural network. Low intra-cluster distance values implies on more and more similar patterns at the same cluster.

In order to validate the equations 8 and 9 , experiments have been carried out using data sets where the ideal value for the vigilance parameter is known. The adopted data sets were Iris and Spanning Tree Blake and Merz, 1998 with the respective ideal vigilance values of 0.99 Vicentini, 2002; Vicentini and Romero, 2003 and 0.95 Fausett, 1994. The figures 3 and 4 show the results of the intra and inter-cluster distances to $\rho \in[0,1]$. Observing the figure 3 , 
it may be concluded that the ideal vigilance is from 0.98 to 0.99 , which is a value very close to the Iris' ideal one. The figure 4 allows the conclusion that the ideal value for the Spanning Tree data set is around 0.95, what corresponds to the result obtained on Fausett, 1994.

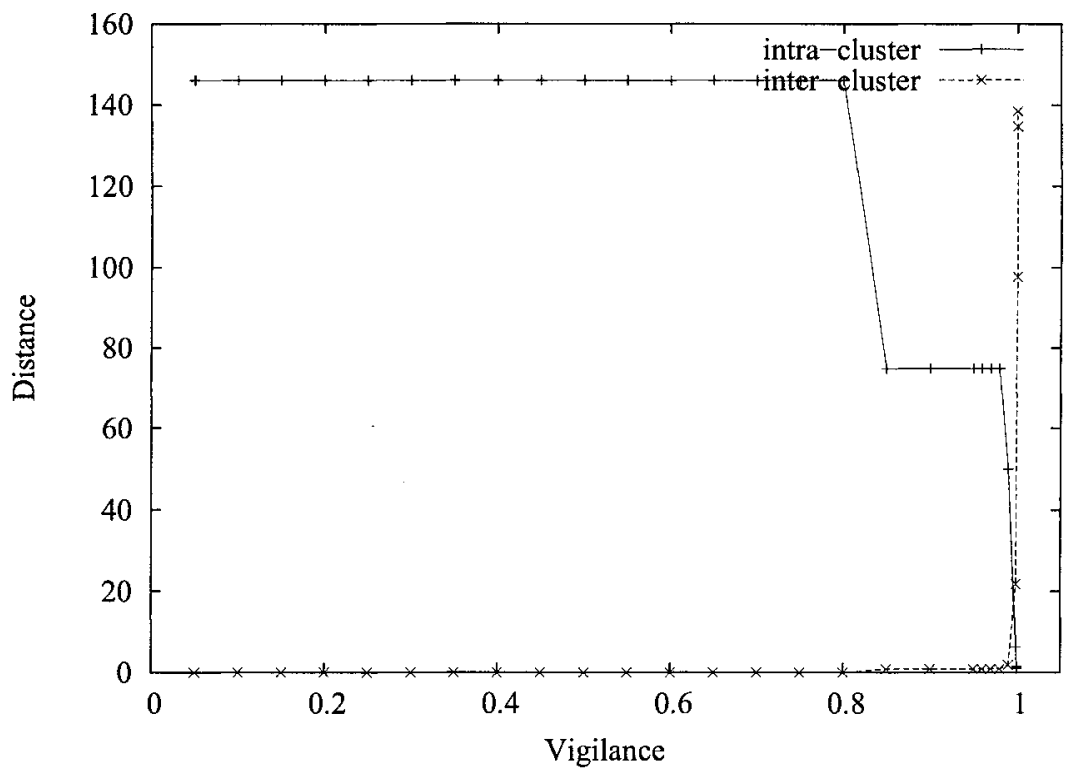

Figure 3. Iris Experiment: Intra and inter-cluster distance variance

The results of the experiments carried out on Iris and Spanning Tree data sets allows the validation of the results obtained through the distance equations that are used to automatically define the vigilance values, which were previously defined in a manual basis. Such equations are used to define the best vigilance values for the experiments presented as follows.

\section{Experiments}

In order to demonstrate the application of the proposed model, experiments have been carried out using the following data sets: The Dataset ${ }^{2}$ and 20 Newsgroup ${ }^{3}$.

The first experiment was carried out using part of the The Dataset data set, which contains $\mathrm{html}$ pages ranked according to the table 4.100 documents of the Programming Languages - $\mathrm{C} / \mathrm{C}++$ type and other 100 from the Science Biology type were evaluated. Out of these documents, the tags $\mathrm{html}{ }^{4}$ were removed. 


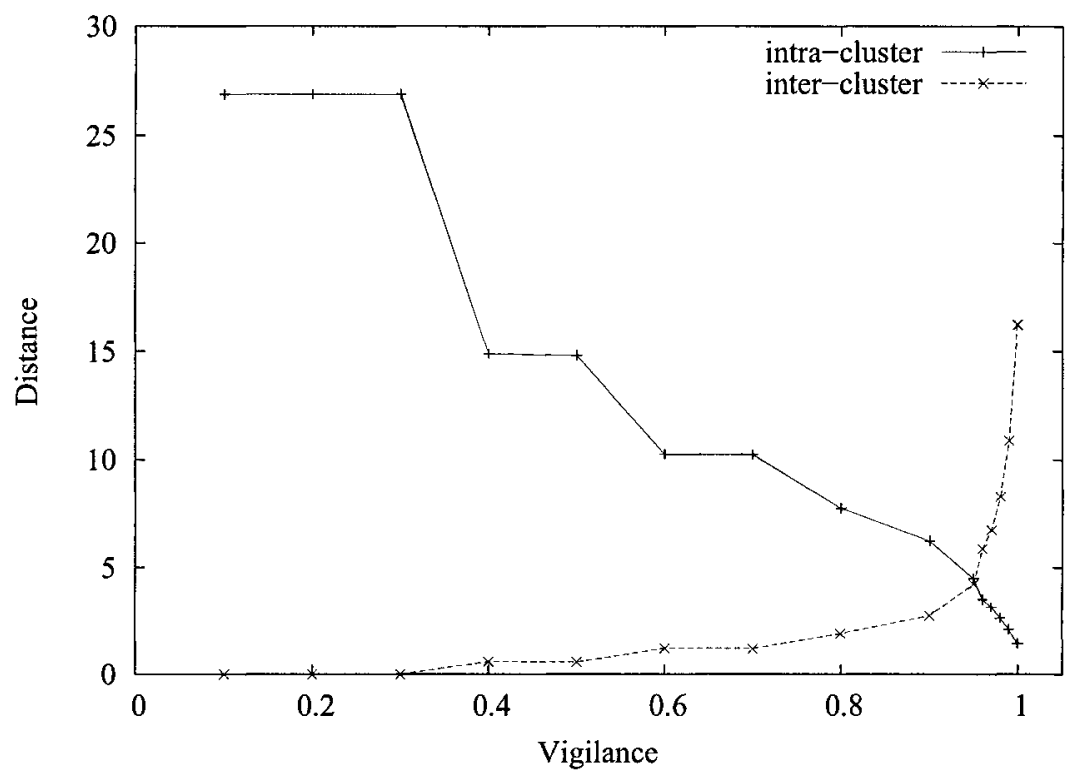

Figure 4. Spanning Tree Experiment: Intra and inter-cluster distance variance

Table 4. Categories of The Dataset

\begin{tabular}{ccc}
\hline Categories & Subject & Number of Documents \\
\hline \hline Commercial Banks & Banking \& Finance & 1000 \\
\hline Building Societies & Banking \& Finance & 1000 \\
\hline Insurance Angencies & Banking \& Finance & 1000 \\
\hline Java & Programming Languages & 1000 \\
\hline C/C++ & Programming Languages & 1000 \\
\hline Visual Basic & Programming Languages & 1000 \\
\hline Astronomy & Science & 1000 \\
\hline Biology & Science & 1000 \\
\hline Soccer & Sport & 1000 \\
\hline Motor Sport & Sport & 1000 \\
\hline General Sport & Sport & 1000 \\
\hline
\end{tabular}

It may be noted that some $C / C++$ and Biology documents were arranged on a same cluster. This fact has motivated the analysis of the file contents, which has proved that some texts of the Biology category had many keywords similar to the ones found on the $\mathrm{C} / \mathrm{C}++$ texts. In some cases there were programming language commands within the Biology texts. This allows the conclusion that the human classification made on these data sets does not deal with possible subject intersections contained on them. The classification results of the neural 
network for $\rho \in[0,1]$ were evaluated by the equations 8 and 9 , from which were generated the functions shown on figure 5 . When making the regression of such functions, the equations on the inter and intra-cluster distances presented on table 5 were obtained. The equations were equalized in order to find out the point where both have the same distance. On this case, the ideal $\rho$ is equal to 0.13568 .

Found the ideal vigilance parameter, the document feature vectors were once again submitted to the neural network, which, then, classified them. Out of this classification were extracted the most relevant word radicals and their respective significances shown on table 6 .

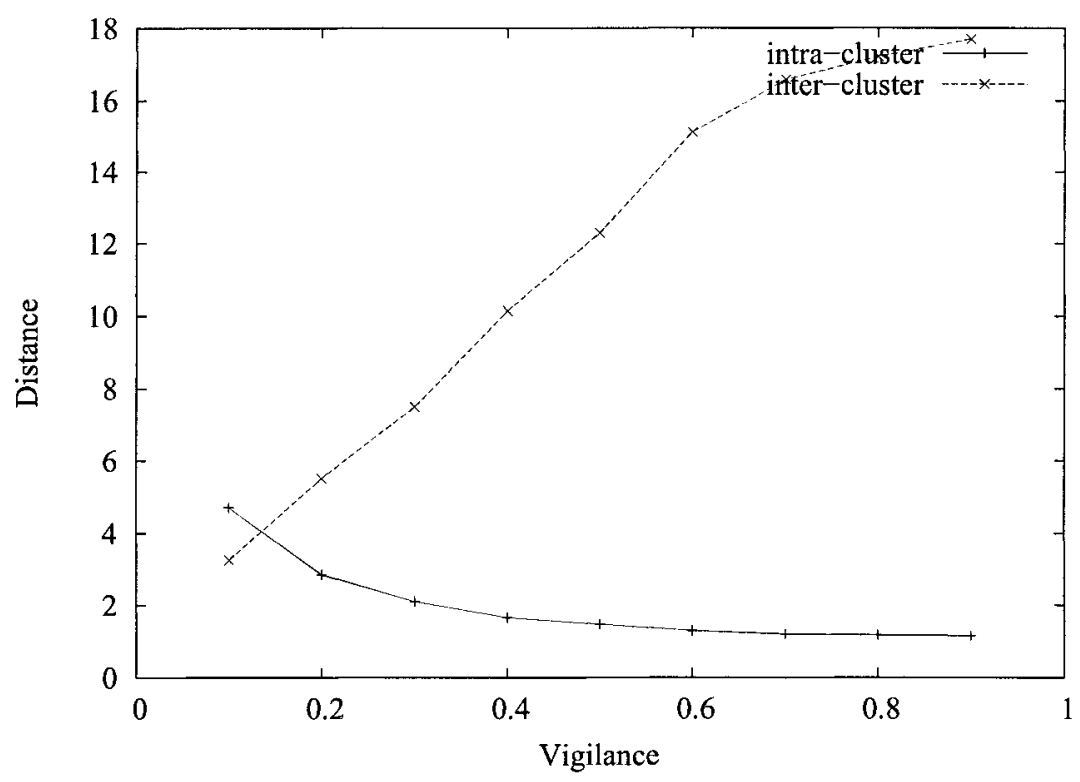

Figure 5. The Dataset Experiment: Intra and inter-cluster distance variance

Table 5. The Dataset Experiment: Equations of Distance

\begin{tabular}{ccc}
\hline Distance & Equation & $R^{2}$ \\
\hline Intra-cluster & $-19.35 x_{3}+38.34 x_{2}-25.11 x+6.72$ & 0.989 \\
\hline Inter-cluster & $-35.70 x_{3}+40.28 x_{2}+10.02 x+1.96$ & 0.998 \\
\hline
\end{tabular}

The second experiment has been carried out on part of the 20 Newsgroup data set, which is composed of documents from a discussion list ranked according to the table 7. Out of these documents were removed tags html ${ }^{5}$. After this stage was completed, 300 documents were randomly distributed into three 
Table 6. The Dataset Experiment: Radicals and Significance

\begin{tabular}{lcl}
\hline Cluster & Patterns & Main Radicals e Significance $(\%)$ \\
\hline \hline$C_{0}$ & 15 & size $=5.08$, deand $=5.16$, e0001 $=7.07$ \\
\hline$C_{1}$ & 42 & site $=3.33$, topic $=3.51$, web $=6.31$ \\
\hline$C_{2}$ & 14 & lantra $=6.15$, indexold $=8.22$, free $=30.78$ \\
\hline$C_{3}$ & 2 & place $=3.35$, vietnames $=12.98$, glossari $=13.34$ \\
\hline$C_{4}$ & 2 & global $=44.86$, yellow $=46.41$ \\
\hline$C_{5}$ & 5 & vni $=2.43$, servic $=3.31$, net $=7.72$ \\
\hline$C_{6}$ & 3 & lo $=5.99$, chile $=6.45$, espectador $=10.22$ \\
\hline$C_{7}$ & 7 & offic $=4.59$, sharewar $=4.74$, librar $i=5.19$ \\
\hline$C_{8}$ & 2 & parentmenu $=4.09$, indexof $=4.78$, pleas $=5.77$ \\
\hline$C_{9}$ & 1 & columbia $=60.70$ \\
\hline$C_{10}$ & 4 & line $=4.21$, hall $=6.32$, korean $=11.49$ \\
\hline$C_{11}$ & 4 & aquariu $=4.50$, lantra $=4.66$, mutipl $=5.35$ \\
\hline$C_{12}$ & 6 & pro $=5.84$, systran $=6.80$, serbian $=12.01$ \\
\hline$C_{13}$ & 4 & fear $=4.87$, daili $=4.92$, sovinformbureau $=15.38$ \\
\hline$C_{14}$ & 3 & consortium $=5.74$, explor $=7.65$, coder $=8.49$ \\
\hline$C_{15}$ & 6 & number $=6.56$, shredder $=7.59$, engin $=7.59$ \\
\hline$C_{16}$ & 12 & asm $=3.01$, templat $=3.52$, fear $=7.80$ \\
\hline$C_{17}$ & 4 & group $=4.90$, usenet $=9.80$, usafa $=14.70$ \\
\hline$C_{18}$ & 6 & lumpin $=7.09$, encapsul $=7.09$, seri $=8.83$ \\
\hline$C_{19}$ & 7 & side $=4.41$, backslash $=5.79$, abil $=6.16$ \\
\hline$C_{20}$ & 25 & transliter $=5.45$, improv $=13.60$, sector $=13.60$ \\
\hline$C_{21}$ & 9 & latvian $=4.49$, chines $=4.49$, includ $=5.99$ \\
\hline$C_{22}$ & 3 & mark $=10.81$, complet $=13.19$, todo $=15.60$ \\
\hline$C_{23}$ & 2 & impli $=2.86$, freewar $=11.41$, screenshot $=19.98$ \\
\hline$C_{24}$ & 1 & clock $=4.38$, atl $=4.53$, skin $=7.32$ \\
\hline$C_{25}$ & 7 & mile $=4.88$, impli $=8.44$, remeb $=8.88$ \\
\hline$C_{26}$ & 4 & mesmer $=3.67$, gun $=7.34$, alphabet $=8.35$ \\
\hline & & \\
\hline
\end{tabular}

categories: alt.atheism, rec.motorcycles and sci.space. The stopwords and the radicals were removed and their respective frequency counting was made.

The results of the intra and inter-cluster distances to $\rho \in[0,1]$ are presented on the figure 6 . This experiment included classification cases similar to the previous experiment, where documents from distinct categories were arranged in the same cluster. When the content of such documents was better analyzed, it was verified that they presented very similar subjects, although they were arranged in very distinct pre-defined categories.

Out of the results presented on figure 6 , regressions were made and the equations of table 8 were obtained. By equalizing these equations it was possible to obtain the best vigilance parameter for the experiment being conducted, which was $\rho=0.64432$.

After finding the ideal vigilance parameter, the patterns were once again submitted to the neural network, which then classified the documents. Out 
Table 7. Categories of 20 Newsgroup

\begin{tabular}{lc}
\hline Categories & Number of Documents \\
\hline alt.atheism & 1000 \\
\hline Comp.graphics & 1000 \\
\hline comp.os.ms-windows.misc & 1000 \\
\hline comp.sys.ibm.pc.hardware & 1000 \\
\hline comp.sys.mac.hardware & 1000 \\
\hline comp.windows.x & 1000 \\
\hline misc.forsale & 1000 \\
\hline rec.autos & 1000 \\
\hline rec.motorcycles & 1000 \\
\hline rec.sport.baseball & 1000 \\
\hline rec.sport.hockey & 1000 \\
\hline sci.crypt & 1000 \\
\hline sci.electronics & 1000 \\
\hline sci.med & 1000 \\
\hline sci.space & 1000 \\
\hline soc.religion.christian & 1000 \\
\hline talk.politics.guns & 1000 \\
\hline talk.politics.mideast & 1000 \\
\hline talk.politics.misc & 1000 \\
\hline talk.religion.misc & 1000 \\
\hline
\end{tabular}

Table 8. 20 Newsgroup Experiment: Equations of Distance

\begin{tabular}{lccc}
\hline Distance & Equation & $R^{2}$ \\
\hline \hline Intra-cluster & $9054.93 x_{4}-21057.62 x_{3}+17455.76 x_{2}-6130.96 x+796.36$ & 0.983 \\
\hline Inter-cluster & $3497.84 x_{4}-5497.00 x_{3}+3019.84 x_{2}-629.60 x+40.24$ & 0.995 \\
\hline
\end{tabular}

of this classification were extracted the most relevant word radicals and their respective significances were presented on tables 9 and 10.

The classification generated by the ART-2A neural network architecture for the two experiments has provided a group of similar documents in a same cluster. Moreover, it was possible to extract the $C$ attribute set (or word radicals) and its respective significances from each group. Such information allows the creation of a document database, which is indexed through the $C$ attribute set. Over this database it is possible to make searches by means of keywords. As a result, documents are presented and they may be arranged according to their respective significance levels, based on the searching words defined by the users. 
Table 9. 20 Newsgroup Experiment: Radicals and Significance

\begin{tabular}{|c|c|c|}
\hline Cluster & Patterns & Main Radicals and Significance (\%) \\
\hline$\overline{\overline{C_{0}}}$ & 309 & cleveland $=11.11$, path $=12.12$, usenet $=24.24$ \\
\hline$\overline{C_{1}}$ & 5 & post $=15.80$, cleveland $=16.62$, research $=16.62$ \\
\hline $\mathrm{C}_{2}$ & 5 & reston $=8.16$, in $=10.20$, howland $=14.28$ \\
\hline$\overline{C_{3}}$ & 1 & jame $=7.51$, srv $=10.04$, messag $=12.54$ \\
\hline$C_{4}$ & 2 & juri $=8.76$, felder $=11.66, d a=17.50$ \\
\hline$C_{5}$ & 4 & $n o c=10.93,3$ noc $=10.93$, inc $=14.77$ \\
\hline$\overline{C_{6}}$ & 2 & newsgroup $=10.20$, harvard $=14.30, c m u=26.56$ \\
\hline$\overline{C_{7}}$ & 3 & mark $=7.70$, line $=15.41$, wisc $=33.42$ \\
\hline$\overline{C_{8}}$ & 2 & $d a=13.29$, wisc $=17.72$, reston $=22.16$ \\
\hline$\overline{C_{9}}$ & 9 & mccullou $=11.19$, edu $=14.95$, cmu $=14.95$ \\
\hline$C_{10}$ & 18 & psuvm $=11.35$, cmu $=17.02$, watch $=23.53$ \\
\hline$\overline{C_{11}}$ & 4 & $d o n=7.97, s r v=9.22,10 s r v=15.94$ \\
\hline$\overline{C_{12}}$ & 4 & $a p r=7.22$, humbl $=7.85$, harvard $=10.83$ \\
\hline$C_{13}$ & 6 & new $=11.10$, cleveland $=13.26, q u i t=17.97$ \\
\hline$C_{14}$ & 18 & research $=7.53$, research $=22.84$, odin $=28.51$ \\
\hline$C_{15}$ & 4 & research $=8.17$, help $=13.63$, phone $=15.11$ \\
\hline$C_{16}$ & 3 & $m e s s a g=13.60, i n c=15.23, a s k=22.67$ \\
\hline$\overline{C_{17}}$ & 4 & technolog $=9.62$, repli $=10.34$, world $=18.11$ \\
\hline$C_{18}$ & 2 & sverdrup $=9.61$, gmt $=10.37$, don $=14.04$ \\
\hline$C_{19}$ & 3 & fun $=17.32$, don $=38.95$ \\
\hline$C_{20}$ & 1 & post $=5.37$, hopper $3=17.84$, watch $=21.81$ \\
\hline$C_{21}$ & 3 & atheism $=8.15$, felder $=10.89$, quit $=14.11$ \\
\hline$C_{22}$ & 5 & god $=7.98, c m u=19.98, d o n=27.67$ \\
\hline$C_{23}$ & 1 & cleveland $=6.47$, obnox $i=17.28$, odin $=28.10$ \\
\hline$C_{24}$ & 3 & don $=8.32$, drop $=8.32$, respond $=31.68$ \\
\hline$C_{25}$ & 1 & david $=5.78$, felder $=11.56$, specif $=17.74$ \\
\hline$C_{26}$ & 3 & subject $=8.94$, path $=12.52$, specif $=18.94$ \\
\hline$\overline{C_{27}}$ & 1 & atheism $=7.30$, phone $=7.51$, zue $=24.99$ \\
\hline$C_{28}$ & 23 & vishnu $=11.20$, oath $=11.82$, sverdrup $=22.41$ \\
\hline$\overline{C_{29}}$ & 7 & affirm $=8.86$, allah $=15.54$, odin $=19.96$ \\
\hline$C_{30}$ & 7 & research $=5.00$, technolog $=10.35$, specif $=18.020$ \\
\hline$\overline{C_{31}}$ & 1 & $i n c=10.88, q u i t=10.88$, ask $=14.50$ \\
\hline$C_{32}$ & 5 & sverdrup $=9.07$, gov $=9.56$, couldn $=15.89$ \\
\hline$C_{33}$ & 3 & obnoxi $=7.34$, sorri $=11.00$, reston $=12.64$ \\
\hline$C_{34}$ & 2 & psuvm $=8.00$, week $=10.52$, could $n=14.02$ \\
\hline$C_{35}$ & 2 & court $=9.42$, reston $=10.80$, testimoni $=14.27$ \\
\hline$\overline{C_{36}}$ & 1 & server $=11.18$, prejud $=11.18$, spbach $=12.38$ \\
\hline$C_{37}$ & 6 & alter $n=9.05$, respond $=10.87$, usenet $=20.90$ \\
\hline$C_{38}$ & 4 & alt $=15.34$, follow $=17.11$, atheism $=25.61$ \\
\hline$C_{39}$ & 3 & center $=8.26$, edu $=14.43$, hel $p=30.92$ \\
\hline$C_{40}$ & 1 & lewi $=8.48$, reston $=9.57$, sorri $=11.44$ \\
\hline$C_{41}$ & 75 & newsgroup $=12.98$, dave $=16.36$, respond $=21.84$ \\
\hline$\overline{C_{42}}$ & 7 & harvard $=9.17$, new $=9.17$, affirm $=32.72$ \\
\hline$\overline{C_{43}}$ & 19 & altern $=34.26$, messag $=50.59$ \\
\hline$C_{44}$ & 44 & $e d u=10.60$, hopper $3=21.21$, spbach $=21.21$ \\
\hline$C_{45}$ & 1 & drop $=13.82$, request $=20.74$, line $=24.47$ \\
\hline
\end{tabular}


High Performance Computational Science and Engineering

Table 10. 20 Newsgroup Experiment: Radicals and Significance

\begin{tabular}{lll}
\hline Cluster & Patterns & Main Radicals and Significance (\%) \\
\hline $\bar{C}_{46}$ & 6 & nasa $=25.23$, case $=41.12$ \\
\hline$C_{47}$ & 5 & newsgroup $=27.90$, relev $=29.49$ \\
\hline$C_{48}$ & 11 & testimoni $=12.01$, line $=20.40$, respond $=24.02$ \\
\hline$C_{49}$ & 6 & cwr $u=8.23$, in $=16.43$, prejud $=31.16$ \\
\hline$C_{50}$ & 9 & juri $=28.81$, lewi $=44.80$ \\
\hline$C_{51}$ & 1 & theistic $=9.55$, an $=20.32$, srv $=33.82$ \\
\hline$C_{52}$ & 7 & host $=15.97$, oath $=18.27$, line $=22.79$ \\
\hline$C_{53}$ & 5 & part $=8.90$, center $=17.53$, psuvm $=26.31$ \\
\hline$C_{54}$ & 1 & recit $=14.30$, specif $=21.45$, gmt $=28.79$ \\
\hline$C_{55}$ & 15 & arizona $=8.03$, lewi $=16.65$, requir $=32.09$ \\
\hline$C_{56}$ & 154 & harvard $=14.69$, organ $=22.06$, request $=26.96$ \\
\hline$C_{57}$ & 7 & date $=29.27$, ga $=30.51$ \\
\hline$C_{58}$ & 2 & mccullou $=13.46$, david $=17.92$, newel $=17.92$ \\
\hline$C_{59}$ & 6 & organ $=9.86$, andrew $=12.41$, date $=14.81$ \\
\hline$C_{60}$ & 1 & apr $=11.27$, uug $=13.41$, research $=20.66$ \\
\hline$C_{61}$ & 2 & recommend $=10.60$, ga $=10.60$, nntp $=18.44$ \\
\hline$C_{62}$ & 7 & help $=12.06$, apr $=12.06$, research $=18.08$ \\
\hline$C_{63}$ & 2 & monack $=9.53$, organ $=13.30$, subject $=17.75$ \\
\hline$C_{64}$ & 2 & help $=12.13$, court $=14.55$, atheism $=14.55$ \\
\hline$C_{65}$ & 2 & cmu $=10.99,2$ cmu $=12.84$, requir $=13.74$ \\
\hline$C_{66}$ & 1 & wisc $=8.75$, line $=16.80$, jesu $=17.47$ \\
\hline$C_{67}$ & 1 & felder $=19.17$, world $=19.17$, snake $2=30.79$ \\
\hline$C_{68}$ & 1 & jame $=11.80$, mccullou $=16.86$, path $=35.37$ \\
\hline$C_{69}$ & 1 & fun $=11.29$, newel $=16.92$, center $=27.79$ \\
\hline$C_{70}$ & 2 & help $=8.67$, andrew $=10.70$, jame $=34.80$ \\
\hline$C_{71}$ & 1 & research $=10.14$, mark $=10.50$, center $=11.99$ \\
\hline$C_{72}$ & 5 & psu $=9.82$, mark $=12.63$, center $=14.43$ \\
\hline &
\end{tabular}




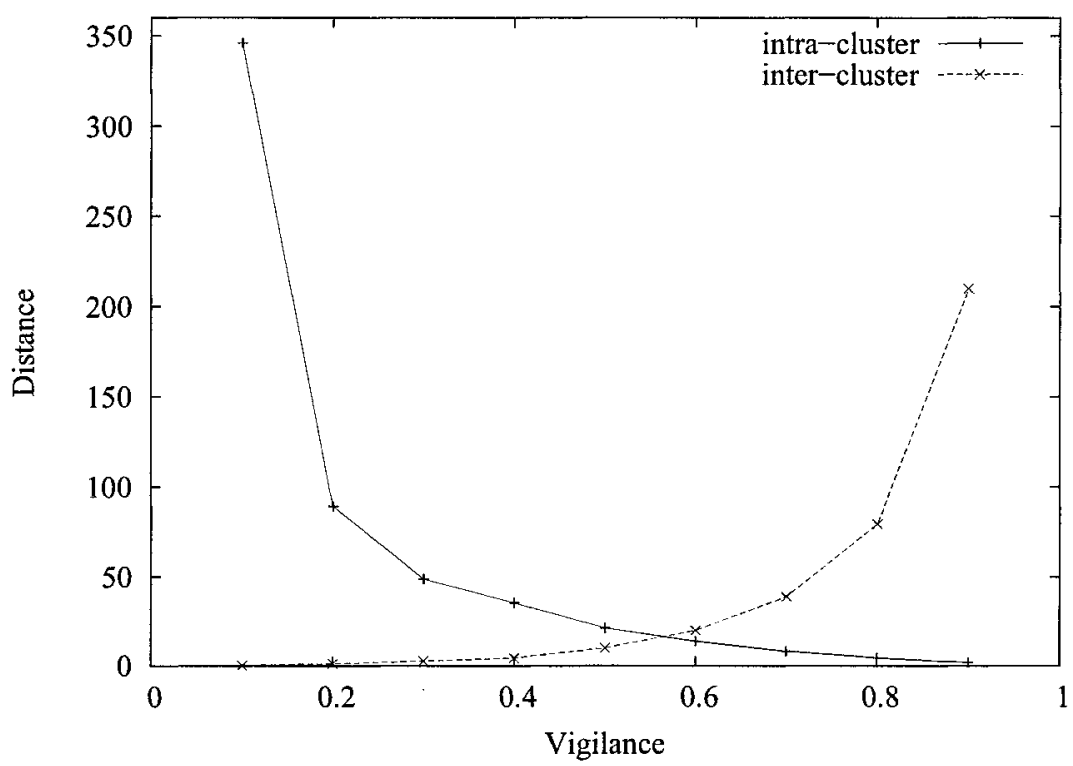

Figure 6. 20 Newsgroup Experiment: Intra and inter-cluster distance variance

\section{Conclusions}

This article presents a new model for automatic text classification. This work has been motivated by the limitations of other classification techniques that require human intervention for the parameterization process Sinka and Corne, 2002; He et al., 2003; Nigam et al., 2000; Blei et al., 2002. The proposed model is composed of the following stages: feature extraction, classification, document labeling and indexing for searching purposes. The feature extraction stage comprehends the word radical frequency counting on the documents. Next stage uses an ART-2A neural network architecture to classify vectors with the word radical frequency on each document. The labeling stage is responsible for extracting the significance level of each word radical at each group generated by the neural network. Such significances are used to index documents, what supports the next stage that comprehends the document search. The main contributions of the proposed model are: proposal for distance measures to automate the $\rho$ vigilance parameter, which is responsible for the classification quality, thus eliminating the human intervention on the parameterization process; proposal for a labeling algorithm that extracts the significance level of each word for each cluster generated by the neural network, and the creation of an automated classification methodology. 
High Performance Computational Science and Engineering

\section{Acknowledgments}

The authors thank to Capes and Fapesp Brazilian Foundations (under the process number 04/02411-9).

\section{Notes}

1. The software source codes to implement the model are available on http://www.icmc.usp.br/mello/outr.html

2. Data set proposed by M.P. Sinka and D.W. Cornei Sinka and Corne, 2002

3. Available on http:/www-2.cs.cmu.edu/afs/cs.cmu.edu/project/theo-20/www/data/news $20 . h t m l$

4. Available on http://www.icmc.usp.br/ mello/outr.html

5. Available on http://www.icmc.usp.br//mello/outr.html 


\section{References}

Bachelder, I., Waxman, A., and Seibert, M. (1993). A neural system for mobile robot visual place learning and recognition. In Proceedings of the 5th Annual Symposium on Combinatorial Pattern Matching, volume 807, pages 198 212, Berlin. Springer.

Blake, C. and Merz, C. (1998). UCI repository of machine learning databases.

Blei, D., Bagnell, J., and McCallum, A. (2002). Learning with scope, with application to information extraction and classification. In Uncertainty in Artificial Intelligence: Proceedings of the Eighteenth Conference (UAI-2002), pages 53-60, San Francisco, CA. Morgan Kaufmann Publishers.

Carpenter, G. A., Gjaja, M. N., Gopal, S., and Woodcock, C. E. (1997). ART neural networks for remote sensing: Vegetation classification from lansat TM and terrain data. IEEE Transactions on Geoscience and Remote Sensing, 35(2):308-325.

Carpenter, G. A. and Grossberg, S. (1988). The ART of adaptive pattern recognition by a selforganizing neural network. Computer, 21:77-88.

Carpenter, G. A. and Grossberg, S. (1989). ART 2: Self-organization of Stable Category Recognition Codes for Analog Input Patterns. Applied Optics, 26(23):4919-4930.

Carpenter, G. A., Grossberg, S., and Rosen, D. B. (1991). ART 2-A: An Adaptive Resonance Algorithm for Rapid Category Learning and Recognition. Neural Networks, 4:4934-504.

Fausett, L. (1994). Fundamentals of Neural Networks. Prentice Hall.

Filippidis, A., Jain, L. C., and Lozo, P. (1999). Degree of familiarity ART2 in knowledge-based landmine detection. IEEE Transactions on Neural Networks, 10(1).

Gan, K. and Lua, K. (1992). Chinese character classification using adaptive resonance network. Pattern Recognition, 25:877-888.

Gokcay, E. and Principe, J. (2000). A new clustering evaluation function using renyi's information potential.

He, J., Tan, A.-H., and Tan, C.-L. (2003). Modified art 2 a growing network capable of generating a fixed number of nodes. IEEE Transactions on Neural Networks. In press.

Keyvan, S. and Rabelo, L. C. (1992). Sensor signal analysis by neural networks for surveillance in nuclear reactors. IEEE Transactions on nuclear science, 39(2).

Nigam, K., McCallum, A. K., Thrun, S., and Mitchell, T. M. (2000). Text classification from labeled and unlabeled documents using EM. Machine Learning, 39(2/3):103-134.

Pappas, T. (1989). The Joy of Mathematics. Wide World Publishing.

Pierre, J. (2000). Practical issues for automated categorization of web pages.

Senger, L. J., de Mello, R. F., Santana, M. J., Santana, R. H. C., and Yang, L. T. (2004). An online approach for classifying and extracting application behavior on linux. In Yang, L. T. and Guo, M., editors, High Performance Computing: Paradigm and Infrastructure. John Wiley \& Sons. 
Sinka, M. and Corne, D. (2002). A large benchmark dataset for web document clustering.

Ultsch, A. (1993). Self-organising neural networks for monitoring and knowledge acquisition of a chemical process. In Proceedings of ICANN-93, pages 864-867.

Vicentini, J. F. (2002). Indexao e recuperao de informaes utilizando redes neurais da famlia art. Master's thesis, Instituto de Cincias Matemticas e de Computao da Universidade de So Paulo.

Vicentini, J. F. and Romero, R. A. F. (2003). Utilizacao de redes neurais da famlia art para indexao e recuperaqo de informaes. In 4th Congress of Logic Aplied to Technology, pages 195-202.

Vlajic, N. and Card, H. C. (2001). Vector quantization of images using modified adaptive resonance algorithm for hierarchical clustering. IEEE Transactions on Neural Network, 12(5).

Whiteley, J. R. and Davis, J. F. (1993). Qualitative interpretation of sensor patterns. IEEE Expert, 8:54-63.

Whiteley, J. R. and Davis, J. F. (1996). Observations and problems applying ART2 for dynamic sensor pattern interpretation. IEEE Transactions on Systems, Man and Cybernetics-Part A: Systems and Humans, 26(4):423-437. 